\title{
Was war die Erlanger Handlungspsychologie? Eine dreiteilige Skizze
}

\author{
Carlos Kölbl
}

Eingegangen: 11. Oktober 2021 / Angenommen: 13. November 2021 / Online publiziert: 22. Dezember 2021

(C) Der/die Autor(en) 2021

Zusammenfassung Im Zentrum des vorliegenden Beitrags steht die Frage danach, was die Erlanger Handlungspsychologie war. Der Versuch einer Antwort erfolgt in drei Schritten. Erstens werden Genese, Entwicklung und Etablierung der Erlanger Handlungspsychologie, zweitens ihr Handlungsbegriff sowie Formen der Handlungserklärung und drittens Schwierigkeiten und Alternativen des Empiriebezugs nachgezeichnet. Die skizzenhafte und pointierte Rekonstruktion dieses Ansatzes erfolgt dabei immer auch mit Blick auf andere handlungspsychologische Forschungsprogramme, um seine Spezifika besser herausarbeiten zu können.

Schlüsselwörter Kulturpsychologie $\cdot$ Psychologiegeschichte ·

Wissenschaftstheorie $\cdot$ Konstruktivismus $\cdot$ Methodologie

Carlos Kölbl ( $\square)$

Lehrstuhl für Psychologie, Kulturwissenschaftliche Fakultät, Universität Bayreuth, Bayreuth, Deutschland

E-Mail: carlos.koelbl@uni-bayreuth.de 


\title{
Erlangen Psychology of Action in hindsight. A tripartite sketch
}

\begin{abstract}
The present contribution focusses on the question of what the Erlangen Psychology of Action was. An attempt to answer this question is made in three steps: First, the genesis, development, and establishment of the Erlangen Psychology of Action, second, its notion of action and forms of explaining actions, and, third, difficulties and alternatives with reference to empirical data are traced. The sketchy and pointed reconstruction of the Erlangen Psychology of Action is always contrasted with other psychologies of action, in order to be able to work out its specific features.
\end{abstract}

Keywords Cultural psychology · History of psychology $\cdot$ Philosophy of science · Constructivism $\cdot$ Methodology

Handlungspsychologisches Denken und Forschen basiert auf spezifischen anthropologischen Vorannahmen. Zu diesen Vorannahmen oder zu diesem Menschenbild gehört die Vorstellung vom Menschen als eines prinzipiell reflexiven, autonomen (in welch mehr oder weniger eng gesteckten Grenzen auch immer) und mit seiner sozialen wie natürlichen Umwelt interagierenden Wesens. Damit wird eine Grenze $\mathrm{zu}$ Theorieströmungen gezogen, in denen Menschen vorzugsweise als Organismen betrachtet werden, die von externen oder internen Reizen, Trieben oder Instinkten gesteuert werden. Die Handlungspsychologie erlebte im deutschsprachigen Raum ab den 1970er-Jahren einen deutlichen Aufschwung (s. etwa Thomas 1976; Groeben und Scheele 1979; Heckhausen 1980). Die Gründe hierfür sind kaum auf einen Nenner zu bringen, die entsprechenden Forschungsprogramme und die Forschungstraditionen, auf die sie sich bezogen, ebenso wenig. Aufschwung sowie theoretische und empirische Ausdifferenzierungen fanden auch vor dem Hintergrund eines gesteigerten multi-, inter- und transdisziplinären Interesses statt, was etwa an den von Hans Lenk herausgegebenen Sammelbänden deutlich wird (Lenk 1977-1984; für die Psychologie von besonderem Interesse ist Band III ,Verhaltenswissenschaftliche und psychologische Handlungstheorien", mit einem ersten und einem zweiten Halbband 1981 bzw. 1984). Zu denjenigen Wissenschaftlern, die sich daranmachten, Psychologie dezidiert als Handlungspsychologie zu betreiben, gehörte in besonderer Weise Hans Werbik und seine am Lehrstuhl für Psychologie II am Institut für Psychologie der Friedrich-Alexander-Universität Erlangen-Nürnberg angesiedelte Arbeitsgruppe.

Was für eine Handlungspsychologie war das, die in Erlangen initiiert und betrieben wurde? Was hatte sie gemein mit anderen handlungspsychologischen Forschungsprogrammen, was waren ihre Spezifika? Diese Fragestellung steht im Zentrum des vorliegenden Beitrags. Dabei soll keine Psychologiegeschichte zu archivarischen Zwecken betrieben, sondern eine (aus Platzgründen notwendigerweise lediglich skizzenhafte und pointierte) Rekonstruktion vorgenommen werden, die auf die Gegenwart und Zukunft zielt. Eine wie auch immer geartete Vollständigkeit der Darstellung wird selbstverständlich nicht beansprucht. Die Handlungspsychologie, wie sie sich als institutionalisierter Arbeitszusammenhang am Erlanger Institut für Psychologie gestaltete, ist ein abgeschlossenes Kapitel. Ein Kapitel, dessen Lektüre 
sich jedoch - das ist jedenfalls die Annahme der folgenden Ausführungen und soll sich eben dort auch zeigen - nach wie vor lohnt.

\section{Genese, Entwicklung und Etablierung}

Die Berufung von Hans Werbik als zweiter Lehrstuhlinhaber (neben Walter Toman) an das Erlanger Institut für Psychologie im Jahr 1973, wo er bereits seit 1970 als Akademischer Rat forschte und lehrte, stellt die Grundlage für die Entstehung der Erlanger Handlungspsychologie dar. Schon seine Beiträge zur Aggressionsforschung (Werbik 1971, 1974) sind handlungspsychologisch ausgerichtet. 1976 erscheinen sodann seine „Grundlagen einer Theorie sozialen Handelns“ in zwei Teilen in der damals für grundlagentheoretische Auseinandersetzungen noch sehr offenen Zeitschrift für Sozialpsychologie (Werbik 1976a, b). 1978 - gewissermaßen als erster Kulminationspunkt - veröffentlicht Werbik den Band „Handlungstheorien“ in der von Theo Herrmann, Werner Tack und Franz Weinert herausgegebenen Reihe „Kohlhammer Standards Psychologie. Basisbücher und Studientexte“ (Werbik 1978) ${ }^{1}$.

In diesen frühen Arbeiten wird mindestens zweierlei deutlich: das Anliegen, Psychologie als eine Wissenschaft zu betreiben, die gesellschaftlich und praktisch relevant ist, sowie der Versuch, mit einer integrativen Handlungspsychologie der Fragmentierung der Psychologie in eine Fülle kleiner und Kleinsttheorien nebst entsprechenden Myriaden an nicht selten auch widersprüchlichen experimentellen und sonstigen empirischen Befunden entgegenzuwirken. Flankierende und mehr noch darauffolgende Beiträge - teilweise gemeinsam mit seinen Mitarbeiterinnen und Mitarbeitern geschrieben - befassen sich mit wissenschaftstheoretischen Grundlagen der Psychologie aus der Perspektive der Erlanger Schule des Konstruktivismus (manchmal auch ,konstruktive Wissenschaftslehre“ genannt; Toebe et al. 1977), loten Grenzen einer nomologischen Auslegung von Handlungstheorien aus (Werbik 1984) und eruieren alternative Möglichkeiten der erfahrungsbasierten Theoriebildung (Aschenbach et al. 1983; Werbik 1987). Diese und andere Auseinandersetzungen führen nicht zuletzt dazu, Handlung als Bindeglied zwischen Individuum und Kultur, Handlung notwendigerweise als kulturell imprägniert, mithin Handlungsund Kulturpsychologie als unauflösliches Junktim zu betrachten (Werbik 1985a, 1986; Zitterbarth 1988; Straub 1999). Die eben angedeuteten Interessen kommen im Bereich der Lehre insbesondere in den einander ablösenden Vertiefungsfächern „Verhaltenstheorie“ (bis 1979), „Theoretische Systeme der Psychologie“ (bis 1985) sowie „Handlungs- und Kulturpsychologie“ (bis Werbiks Emeritierung 2006) zur Geltung (vgl. die entsprechenden Vorlesungsverzeichnisse der Friedrich-AlexanderUniversität Erlangen Nürnberg vom Sommersemester 1979 bis zum Wintersemester 2005/6).

Dieses überaus kurze Narrativ bedarf zumindest einer wesentlichen Ergänzung, ohne die es sich allzu glatt lesen würde. Die angesprochene Genese, Entwicklung

\footnotetext{
1 Der instruktive Einführungsband „Handlungspsychologie“ von Kaiser und Werbik aus dem Jahr 2012 kann (auch) als eine Art aktualisierte Fassung der Monographie von 1978 gelesen werden (Kaiser und Werbik 2012). Für neuere konzise einführende Texte s. Kaiser und Werbik (2018) sowie Straub (2020).
} 
und Etablierung vollzieht sich nämlich keineswegs unproblematisch, am besten noch im Sinne einer linearen wissenschaftsimmanenten Fortschrittsgeschichte. Vielmehr bindet einer der Hauptprotagonisten der Erlanger Handlungspsychologie, eben Hans Werbik, diese in einem Vortrag beim 35. Kongress der Deutschen Gesellschaft für Psychologie in Heidelberg gerade auch an das Erleben existenzieller Krisen (Werbik 1986). Dies geht über die Unzufriedenheit mit dem Zustand psychologischer Theoriebildung deutlich hinaus, wie sie bei der Hinwendung zur Handlungspsychologie, aber auch bei einem gemeinsam mit Heinz Jürgen Kaiser herausgegebenen Sammelwerk zur kritischen Sichtung der Sozialpsychologie zu Beginn der 1980erJahre artikuliert worden war (Werbik und Kaiser 1981). Vielmehr geht es um das Erleben von Sinnhaftigkeit im eigenen wissenschaftlichen Handeln bzw. um den als schmerzlich empfundenen Mangel an solch einem sinnhaften Erleben. Es ist speziell im Angesicht gravierender, ,existentielle[r] Probleme von Menschen“, die ,immer auf Kulturprobleme hinweisen und umgekehrt" (Werbik 1986, S. 1) - wie das als Beispiel gebrachte nukleare Wettrüsten -, evident, dass Werbik die Defizite seiner Wissenschaft, zu eben diesen Problemen etwas Wesentliches zu sagen, als geradezu niederschmetternd empfindet. Sein eigenes Krisenerleben resultiert aus dieser Erfahrung und dem Problem, das sich ergibt, wenn man dennoch als wissenschaftlich tätiger Psychologe zu den angesprochenen Problemen arbeiten wolle:

„Ein Weg aus einer solchen existentiellen Krise kann darin bestehen, daß man sich als Wissenschaftler den Kulturproblemen stellt und versucht, die subjektive Seite dieser Kulturprobleme zu explizieren. Versuche ich dies, dann entsteht für mich unmittelbar ein weiteres gravierendes Dilemma: Entweder ich halte mich an die herrschenden Auffassungen und methodischen Standards unseres Faches. Dann muß ich sehen, daß die wissenschaftliche Psychologie zur Explikation der subjektiven Seite von Kulturproblemen fast nichts beiträgt. Versuche ich hingegen, für die Entwicklung eines Problembearbeitungsansatzes Anregungen zu suchen, wo immer ich sie finde, bin ich außerhalb des Akzeptanzrahmens der psychologischen Wissenschaft und ich muß dann sehen, daß meine Arbeit von der Zunft nicht als Wissenschaft anerkannt wird“" (ebd.; Herv. i. Orig.).

Die Handlungs- und Kulturpsychologie wird im weiteren Verlauf des Vortrags als Möglichkeit vorgestellt, Psychologie weiterhin als Wissenschaft zu betreiben, aber eben jenseits des nomologischen Modells, und mit ihr zur Explikation der subjektiven Seite von Kulturproblemen beizutragen (ebd., S. 4 ff.). Auch ruft Werbik Kolleginnen und Kollegen auf, die aus dem üblichen Modell, Psychologie zu betreiben, ausscheren wollen, sich zu organisieren (ebd., S. 11). Im selben Jahr gründet er denn auch gemeinsam mit anderen die „Gesellschaft für Kulturpsychologie“ in Salzburg.

Was hier mit wenigen Strichen nachgezeichnet wird, ist für Handlungspsychologinnen und Handlungspsychologen nicht die Regel, aber auch keine bloße Idiosynkrasie. Richtet man sein Augenmerk allein auf den deutschsprachigen Raum im Kontext der US-amerikanischen Psychologie wäre etwa an Jerome Bruner oder Michael Cole zu denken -, treten sogleich zwei weitere Psychologen ins Blickfeld: Ernst Boesch (Saarbrücken) und Klaus Holzkamp (West-Berlin). Auch für deren Arbeiten ist der Begriff des menschlichen Handelns zentral, und es gibt von ihnen ebenfalls so etwas wie Krisenerzählungen in der Perspektive der ersten Person. 
Klaus Holzkamp war, bevor er die prägende Figur der Kritischen Psychologie wurde, bereits ein anerkannter Psychologe, der sich bekanntlich insbesondere mit Arbeiten zur Wahrnehmungs- und Sozialpsychologie sowie zu den wissenschaftstheoretischen und methodologischen Grundlagen des Faches einen Namen in der Psychologie gemacht hatte. Im letzten Kapitel seines Buches „Kritische Psychologie. Vorbereitende Arbeiten“, das seinen Bruch mit der „,bürgerlichen“ Psychologie markiert, rekapituliert er nicht zuletzt einen Teil seiner eigenen Biografie als wissenschaftlich tätiger Psychologe in ihrer Verstrickung mit der Geschichte der Psychologie an der FU Berlin vor dem Horizont der Studentenbewegung im Besonderen, der bundesrepublikanischen Geschichte im Allgemeinen (Holzkamp 1972, S. 207-288). In einem späteren Interview mit Hans Schindler finden sich ebenfalls Auskünfte Holzkamps über seine persönliche wissenschaftliche Entwicklung (Holzkamp 1996). Auch er verspürt im Laufe seiner Biografie ein massives Ungenügen an seiner Disziplin. Vereinfachend gesagt, speist sich dieses für ihn unter anderem aus ihrem reduktionistischen Menschenbild, ihrer variablenpsychologischen und „,kontrollwissenschaftlichen“ Anlage, ihrer Dienstbarkeit für die Interessen ,der Herrschenden" und der Verfehlung ihres eigentlichen Gegenstandes. Im Gegenzug ist Holzkamp an der Schaffung einer Psychologie gelegen, die menschliches Handeln zu einem ihrer Grundbegriffe macht, historisch in einem weiten Sinne angelegt ist und emanzipatorischen Ansprüchen genügt. Letztere sind für ihn mit einer dezidierten kommunistischen Parteinahme verknüpft, die allerdings in seinem letzten Buch zur Thematik menschlichen Lernens kaum noch - falls überhaupt - auszumachen ist (Holzkamp 1993).

Von Ernst Boesch gibt es mehrere autobiografische Texte, in denen er seine Abwendung von der ,traditionellen“ Psychologie und seine allmählich sich vollziehende Erarbeitung einer symbolischen Handlungs- und Kulturpsychologie nachzeichnet (s. pars pro toto nur den jüngst veröffentlichten Rückblick von Boesch (2021), der 2010 lediglich als Privatdruck erschienen war). Für ihn sind insbesondere seine Erfahrungen im Zuge eines durch die UNESCO angestoßenen mehrjährigen Forschungsaufenthalts in Thailand von großer, sein wissenschaftliches Tun wie auch sein sonstiges Leben stark verändernder Bedeutung. In Thailand, so ist bei ihm immer wieder zu lesen, seien ihm die Grenzen des üblichen psychologischen Forschens überdeutlich geworden. Vor diesem Aufenthalt war er ein Psychologe, der in der Tradition der Piaget'schen Psychologie stehend zunächst als Schulpsychologe arbeitete, sich mit entwicklungs-, pädagogisch-psychologischen und (test-)diagnostischen Fragestellungen beschäftigte, um später als an die Universität des Saarlandes berufener Professor auch nach seinem Thailandaufenthalt viele Jahre hinweg große Teile der gesamten Psychologie in der Lehre abzudecken. An der ,fremdkulturellen Erfahrung" brach sich für Boesch das herkömmliche theoretische und methodologisch-methodische Instrumentarium seiner Disziplin, was ihn dazu führte, eigene Wege in der Psychologie einzuschlagen. Mitunter legt Boesch aber auch nahe, dass seine kritische Haltung an der üblichen akademischen Psychologie sich nicht erst in Thailand herausbildete, sondern er sich bereits als Student in Genf nach einer „Psychologie der Betroffenheit" sehnte (Boesch 2002), einer Psychologie jenseits der experimentalpsychologischen Laboratorien, die sich lebensweltlichen Problemen in einer beschädigten Welt zuwendet. 
Es ist hier nicht mein Anliegen, eine ausführliche komparative Analyse dreier bis an die Grenze des Zumutbaren gerafft nachgezeichneter krisenhafter wissenschaftlicher Biografien vorzulegen. Im Hinblick auf bemerkenswerte Gemeinsamkeiten und Unterschiede sei lediglich folgendes schematisch festgehalten: Alle drei Psychologen leiden im emphatischen Sinne irgendwann einmal an ihrer Wissenschaft, weil sie sie als höchst defizitär wahrnehmen, was die Bearbeitung wirklicher, lebensweltlicher Probleme anbelangt. Bei Holzkamp und Werbik ist diese Defizitwahrnehmung auch auf eine intensive Beschäftigung mit den wissenschaftstheoretischen und methodologisch-methodischen Grundlagen des Faches zurückzuführen, wobei im Falle Holzkamps vor seiner marxistischen Wende Hugo Dinglers Einfluss, im Falle Werbiks die Bedeutung der Erlanger Konstruktivisten Paul Lorenzen, Wilhelm Kamlah und Oswald Schwemmer besonders groß ist. Dem empfundenen Ungenügen begegnen wiederum alle drei mit der Erarbeitung bzw. Weiterentwicklung ganz unterschiedlicher psychologischer Handlungstheorien, die sich gerade auch auf die Überwindung individuozentrierter Engführungen psychologischen Denkens und Forschens richten. Für diese Überwindung steht bei Werbik und Boesch der Konnex von Handlungs- und Kulturpsychologie ${ }^{2}$, bei Holzkamp die Leitvorstellung einer marxistisch fundierten Subjektwissenschaft mit entsprechender gesellschaftstheoretischer Rahmung. Kulturpsychologie wird für Boesch insbesondere vor dem Hintergrund seiner ,fremdkulturellen“ Erfahrungen in Thailand virulent, wohingegen Werbik zur Kulturpsychologie über seine Kritik an einer Einhegung handlungspsychologischen Denkens in einer nomologischen Psychologie und seine Betroffenheit durch existenzbedrohende Kulturprobleme findet. Solch ,existenzbedrohende Kulturprobleme", wie die von Werbik ins Feld geführte Bedrohung durch das nukleare Wettrüsten, der er sich auch zum Teil mit seinen Mitarbeitern in einigen seiner Schriften eigens widmet (z. B. Straub et al. 1987), spielen auch für Holzkamp eine große Rolle, wobei seine Interpretation solcher Phänomene und ihrer ,psychologischen Seite“ deutlich anders ausfällt, weil sie wesentlich vom marxistischen Denken orientiert wird. Alle drei Wissenschaftler nehmen schließlich eine gewisse Marginalisierung im Normalbetrieb der akademischen Psychologie in Kauf. Während allerdings Ernst Boesch trotz großen Lehrstuhls und einer starken an die Universität angebundenen Forschungsstelle, deren Direktor er jahrelang ist, in vielerlei Hinsichten als ein Einzelgänger wirkt, schafft Klaus Holzkamp mit anderen zusammen einen zeitweise beeindruckend großen Arbeitszusammenhang. Für Holzkamp gilt ferner:

„Also ich würde sagen, die Nähe zur kommunistischen Partei [...] war schon eine Art allgemeiner Voraussetzung, um den ganzen Kram in dieser konsequenten Form zu machen. Daß man nicht als isoliertes Individuum rumwirtschaftete, sondern irgendwo sinnvoll in den Gesamtzusammenhang der Arbeiterbewegung [...] integriert war, spielte schon eine große Rolle“. (Holzkamp 1996, S. 577)

\footnotetext{
${ }^{2}$ Dieser Konnex wird bei Boesch im Sinne einer dezidiert symbolischen Handlungs- und Kulturpsychologie erst vergleichsweise spät deutlicher ausbuchstabiert, zumal für eine englischsprachige Leserschaft. Seine einschlägige Monographie erscheint 1991, also erst einige Jahre nach seiner Emeritierung (Boesch 1991). Die Rezeption Boeschs scheint mir im Übrigen etwas zu sehr an der entfalteten symbolischen Handlungs- und Kulturpsychologie orientiert zu sein und dabei ein wenig zu übersehen, dass es auch einen „Boesch davor" gab, der unter anderem an einer Kulturpsychologie der Erziehung und Bildung arbeitete (Kölbl 2020a; knapper: Kölbl 2020b).
} 
Hans Werbik dürfte irgendwo zwischen diesen beiden Polen anzusiedeln sein, sicher aber deutlich mehr auf der Seite von Boesch als von Holzkamp.

\section{Handlungsbegriff und Handlungserklärung}

Wie Handlungspsychologie betrieben wird, hängt trivialerweise in entscheidender Art und Weise davon ab, welchen Begriff der Handlung man zugrunde legt und welche Form oder welche Formen der Handlungserklärung man wählt. Die Erlanger Handlungspsychologie begreift menschliches Handeln in ihrer „Frühzeit“ insbesondere als ein auf Ziele gerichtetes Handeln, das daher auf ein intentionalistisches oder teleologisches Modell der Handlungserklärung angewiesen ist, und bezieht sich dabei unter anderem auf Kurt Lewin, George A. Miller, Eugene Galanter und Karl H. Pribram sowie Gerhard Kaminski in der Psychologie, auf Max Weber in der Soziologie und auf Paul Lorenzen, Wilhelm Kamlah oder Gilbert Ryle in der Philosophie. Absichten, Wünsche, Motive, Vorsätze, Zwecke, Mittel, Pläne, Handlungsgründe, handlungsvorbereitende Kognitionen, Handlungsregulationsprozesse sowie Ziele stellen wichtige Konstituenten eines solchen Handlungsvokabulars dar (Werbik 1976a, b, 1978). Eine wichtige Ergänzung nimmt Gabriele Korthals-Beyerlein in ihrer Dissertation vor, in der sie unter anderem Werbiks Theorie sozialen Handelns im Hinblick auf die Rolle sozialer Normen anreichert (Korthals-Beyerlein 1979, S. 236-247). Günter Aschenbach legt ferner eine rationalitätstheoretisch differenzierte Handlungstypologie vor (Aschenbach 1984, S. 145-176), Ulrike Popp-Baier expliziert autoritäres, autonomes und kommunikatives Handeln als unterschiedliche Typen sozialen Handelns (Popp 1989, S. 49-77). Jürgen Straubs 1994 eingereichte und 1999 veröffentlichte Habilitationsschrift schließlich kann gleichermaßen als Schlusspunkt der Erlanger Handlungspsychologie wie als (wichtige Einsichten der Erlanger Tradition ,,aufhebenden“) Neuanfang gelesen werden (Straub 1999); einen Neuanfang, den unter anderem Barbara Zielke - die letzte Mitarbeiterin an Hans Werbiks Lehrstuhl - mit ihrer Arbeit zu einer ,postkognitivistischen Psychologie“ aufnimmt (Zielke 2004), die der Überwindung der „Halbherzigkeit der kognitiven Wende“" (Zitterbarth und Werbik 1990) dienen soll. In Straubs Schrift werden die bis dahin erarbeiteten handlungstheoretischen Einsichten systematisiert, integriert, weiterentwickelt und vielfach ergänzt, naheliegenderweise in Auseinandersetzung mit benachbarten Handlungspsychologien, aber gerade auch mit der analytischen Philosophie, der Phänomenologie und dem Pragmatismus. Dies geschieht im Zuge der Erarbeitung einer komplexen Typologie, die unterschiedliche Handlungstypen und dazu gehörende Formen der Handlungserklärung umfasst. Im Einzelnen werden zielorientiertes und regelorientiertes Handeln sowie Handeln als und in Geschichte(n) in ihrer Bezogenheit auf das teleologische, das regelbezogene sowie das narrative Modell der Handlungserklärung differenziert (Straub 1999, S. 56-162). Diese Differenzierungen bilden dann auch die Grundlage für seine weitergehenden Ausführungen zur Kulturpsychologie, die er als eine entschieden handlungstheoretisch ausgerichtete Kulturpsychologie anlegt (ebd., S. 162-199).

Die Bindung der Handlungspsychologie an Ziele und ein teleologisches Modell der Erklärung ist auch bei den oben bereits erwähnten Ernst Boesch und Klaus 
Holzkamp sehr deutlich, ohne dass ihre wissenschaftlichen Analysen allein darin aufgehen würden (s. z.B. Boesch 1991; Holzkamp 1993). Jürgen Straub hat in Bezug auf Boesch jüngst in einem Vortrag unterstrichen, dass dessen materiale Analysen häufig auch aus seinem stellenweise etwas engen handlungstheoretischen Instrumentarium ausscherten (Straub 2021a; zu Boeschs symbolischer Handlungstheorie s.a. Kochinka 2020). Das ließe sich wohl auch für Arbeiten im Rahmen der Erlanger Handlungspsychologie zeigen (s. etwa, um nur ein Beispiel zu nennen, die empirischen Analysen in Werbik 1987, S. 141-149, die das intentionalistische Modell übersteigen). Blickt man in Holzkamps letzte Monografie zum Lernen und dort speziell in ihr drittes Kapitel, in dem der Autor die „Grundbegrifflichkeit einer subjektwissenschaftlichen Theorie lernenden Weltaufschlusses“ entfaltet (Holzkamp 1993, S. 175-337), kann man feststellen, dass er Lernen zentral als Lernhandlung begreift und diese wiederum als eine an Zielen orientierte Handlung auffasst. Zugleich liefert er mit der auch phänomenologisch dichten Beschreibung eigener Lernbemühungen, etwa im Hinblick auf ,Schönbergs Orchestervariationen als Lernproblematik", handlungspsychologische Analysen, die sich ebenfalls einer bloß intentionalistischen Beschreibung und Erklärung entziehen (ebd., S. 194-205).

Ein anderer Handlungspsychologe, der im Übrigen auch Arbeiten aus dem Erlanger Konstruktivismus rezipierte, ist Jochen Brandtstädter. Auf ausgewählte Aspekte seines handlungspsychologischen Denkens und Forschens sei nun in kontrastiver Absicht kurz hingewiesen (Brandtstädter 2001; dazu: Kölbl 2003, 2006). Zunächst ist festzuhalten, dass Brandtstädters handlungspsychologisches Denken und Forschen sich insbesondere auf die Ausarbeitung einer handlungstheoretisch orientierten Entwicklungspsychologie richtet, auf die Ausdifferenzierung eines aktionalen Entwicklungsparadigmas. Dabei spielt das intentionale Handeln eine zweifellos bedeutsame Rolle. Und zwar sowohl im Hinblick auf dessen sich allmählich vollziehende Ausbildung und Weiterentwicklung im Lebenslauf als auch im Hinblick auf den „Einsatz“ intentionalen Handelns zur Gestaltung der eigenen Entwicklung. Eine ungebrochene Vorstellung des „Individuums als Produzenten seiner eigenen Entwicklung“ (Lerner und Busch-Rossnagel 1981) ist allerdings Brandtstädters Sache nicht, denn er arbeitet gerade auch die ,prä-, peri- und kontraintentionalen“ Aspekte von Handlungen deutlich heraus und räumt auch „subpersonalen Mechanismen“ bei der Erklärung von Handlungen große Bedeutung ein (s. a. Brandtstädter und Greve 1999). Auch in der (insbesondere ,späteren“) Erlanger Handlungspsychologie erscheint das Subjekt häufig nicht als „Herr im eigenen Haus“ (Freud). Statt allerdings vorzugsweise auf „subpersonale Mechanismen“ zur Erklärung zurückzugreifen, werden in ihr ganz im Sinne einer Handlungs- und Kulturpsychologie - wenn man so möchte - eher „trans-, inter- und extrapersonale“ Hintergründe des Handelns zu explanativen Zwecken exploriert.

\section{Schwierigkeiten und Alternativen des Empiriebezugs}

Handlungspsychologie ist keine Lehnstuhlpsychologie. Die Erlanger Handlungspsychologie war hier keine Ausnahme. Allerdings problematisierte sie den üblichen Empiriebezug der Psychologie. Zunächst hielt sie noch an der Möglichkeit einer 
experimentellen Handlungspsychologie fest (Werbik 1974, 1976a, b), beginnt diese Orientierung allerdings im Zuge eigener desillusionierender einschlägiger experimenteller Studien (Kaiser und Werbik 1977) sowie begleitender wissenschaftstheoretischer und methodologischer Auseinandersetzungen (Toebe et al. 1977; Aschenbach et al. 1983; Werbik 1984) ab Ende der 1970er-Jahre weitgehend aufzugeben zugunsten ,dialogischer“ Verfahren, die an den Relevanzsetzungen der Subjekte ansetzen und in der Auswertung mit interpretativen Analysemethoden operieren (Billmann-Mahecha und Seel 1981; Werbik 1987). ${ }^{3}$ Die wissenschaftstheoretischen und methodologischen Auseinandersetzungen sind - es wurde schon angesprochen - in besonderer Weise von der Erlanger Schule des Konstruktivismus inspiriert worden (z. B. Kamlah und Lorenzen 1967; Kamlah 1972; Lorenzen und Schwemmer 1975). Es ist auch diesen Arbeiten mitgeschuldet, dass in der Erlanger Handlungspsychologie die Frage nach dem Verhältnis von analytischen und empirischen Wahrheiten aufgeworfen und diskutiert wird: „Unter dem Einfluß der konstruktiven Wissenschaftslehre [...] ist die Möglichkeit der ,Analytizität' angeblich empirisch gehaltvoller psychologischer Hypothesen oder Theorien wenigstens ernsthaft und mit einigen Ergebnissen untersucht worden“". (Werbik 1987, S. 134f.; siehe a. Toebe et al. 1977, S. 107 f.; Kempf 1978; Aschenbach et al. 1983, S. 109). Der Einfluss erstreckt sich dabei nicht allein auf die Erlanger Handlungspsychologie, sondern wird - auch dies wurde schon angesprochen - ebenfalls von Jochen Brandtstädter aufgenommen. In Arbeiten zu apriorischen Elementen in psychologischen Forschungsprogrammen (z.B. Brandtstädter 1982, 1984) zeigt er etwa anhand unterschiedlicher Attributionstheorien, dass dort des Öfteren analytische als empirische Zusammenhänge behandelt werden, was zur Erzeugung von Pseudoempirie führt (s. a. Greve 2001).

Im deutschsprachigen Raum beschäftigt sich ebenfalls Klaus Holzkamp prominent mit dieser Thematik und argumentiert, dass häufig Handlungsbegründungen als empirische Zusammenhangsannahmen verkannt würden. Dies versucht er zum einen anhand ausgewählter sozialpsychologischer Theorien nachzuweisen (Holzkamp 1986), zum anderen anhand psychologischer Lerntheorien (Holzkamp 1993). Der Psychologie attestiert er dabei einen ,implizite[n] Begründungsdiskurs“ und ein „nomologisches Selbstmissverständnis“ (ebd., S. 27). Für den Nachweis oder zumindest die Äußerung eines ersten Verdachts, dass eine psychologische Aussage lediglich vermeintlich nomologischen Charakter habe, in Wahrheit aber eine Aussage über Begründungszusammenhänge sei, schlägt Holzkamp vor, zwischen die „Wenn“- und die „Dann“-Komponente einer Aussage die Formel „,vernünftigerwei-

\footnotetext{
3 Auch wenn vielleicht qualitative Verfahren in der Psychologie heutzutage nicht mehr so randständig sein mögen wie noch vor ein paar Jahrzehnten, wird wohl nach wie vor in dieser Disziplin im Großen und Ganzen das Experiment als via regia der Erkenntnis angesehen. Vor diesem Hintergrund dürfte die angesprochene Relativierung der Erkenntnismöglichkeiten psychologischer Experimente ihren Stachel oder dürfte der Titel (nebst Inhalt) eines kurzen Beitrags von Hans Werbik zur ethischen Problematik von Experimenten in der Psychologie seinen provokativen Charakter für nicht wenige wissenschaftlich tätige Psychologinnen und Psychologen auch heute noch immer nicht ganz verloren haben: „Die Versuchspersonen werden vergebens getäuscht. Zur Überflüssigkeit experimenteller Methoden in der Sozialpsychologie“" (Werbik 1985b). Was die Entwicklung qualitativer Methoden angeht, sei hier lediglich auf den seinerzeit innovativen, auf aktuelle psychologische Analysen ikonischen Materials (Straub 2021b, S. 539-595, 2022) vorausweisenden Beitrag von Billmann-Mahecha (1994) hingewiesen, die sich eingehend mit der Erhebung und Auswertung von Zeichnungen (von Kindern) befasste.
} 
se“ einzuschieben. So sei dann eben die Aussage „Wenn es kalt ist, dann zieht man sich warm an“ keine kontingente empirische Aussage, sondern eine ,unexplizierte Begründungsaussage“, was durch das Einschieben der Formel leicht ersichtlich werde: „Wenn es kalt ist, dann zieht man sich vernünftigerweise warm an“ (ebd., S. 34; Herv. i. Orig.). Was Holzkamp hier zunächst an einem einfachen Beispiel demonstriert, wendet er dann auch an, um solche Theorien und Konzepte wie das klassische und das operante Konditionieren, kognitivistische Lerntheorien und andere mehr entsprechend zu kritisieren und $\mathrm{zu}$ reformulieren. Dabei geht es ihm immer um den Nachweis, dass die jeweils von ihm untersuchten psychologischen Theorien in der Terminologie eines „Bedingtheitsdiskurses“ formuliert werden, sie aber tatsächlich „Begründungsmuster“ darstellen würden und daher innerhalb eines „Begründungsdiskurses“, in dem es um Handlungen und Gründe gehe, reformuliert werden müssten und könnten.

Holzkamp entwickelt seine Argumentation zum Nachweis von Begründungsmustern insbesondere in Auseinandersetzung mit den einschlägigen Arbeiten Brandtstädters, aber auch Jan Smedslunds (1991). Ein kurzes Editorial, das Holzkamp in der Zeitschrift für Sozialpsychologie veröffentlicht hat, verweist allerdings bereits 1976 auf die erst später für ihn besonders wichtig werdende Thematik und auch schon auf die versuchsweise einsetzbare Formel, die bei diesem Nachweis hilfreich sein soll. Interessanterweise handelt es sich bei diesem kurzen Text um einen Kommentar zu Hans Werbiks Entwurf einer Theorie sozialen Handelns. Der Kommentar sei hier in Gänze zitiert:

„Was erfährt man eigentlich worüber, wenn man Werbiks ,empirische Hypothesen` (p. 318 ff.) überprüft? Um mir darüber Klarheit zu verschaffen, vergegenwärtige ich mir das Verhalten einer Vp, die diese Hypothesen nicht bestätigt: Eine solche Vp hält sich (vereinfacht formuliert) nicht an ihre eigene Zusage, nur Handlungsmöglichkeiten aus einer bestimmten Liste auszuwählen $(\mathrm{H} 1)$, benutzt trotz ihrer Bereitschaft, ein Ziel zu erreichen, ein ihrer Auffassung nach zur Erreichung dieses Ziels geeignetes Mittel nicht (H 2), akzeptiert ein Mittel zur Zielerreichung nicht, obwohl sie es als für die Erreichung des Ziels hinreichend betrachtet (H 3) und nennt eine bestimmte Handlungsmöglichkeit zur Zielerreichung nicht, obwohl sie deren Geeignetheit zur Errechnung [sic!] des Ziels bei einer anderen Person beobachtet hat (H 4). Ein derartiges Verhalten ist so ,unvernünftig' im alläglichen Sinne, daß es bei einer hinreichend instruierten ,normalen " Vp nicht auftreten wird, womit die Hypothesen ,trivial' sind und ihre Bestätigung ,selbstverständlich ' ist, also unserem Wissen nichts hinzufügt. Die Aufstellung von ,Hypothesen“ täuscht m. E. hier eine empirische Theorie vor, wo in Wirklichkeit nur ein wesentlich tautologisches System mit minimalen empirischen Spielräumen formuliert wurde“. (Holzkamp 1976; Herv. i. Orig.)

Man kann nicht behaupten, dass die von der Erlanger Handlungspsychologie, Jochen Brandtstädter, Klaus Holzkamp, Jan Smedslund und anderen intensiv bearbeitete Problematik breit in psychologischen (oder anderen disziplinären) Fachdis- 
kursen rezipiert und entsprechend berücksichtigt worden wäre - bis heute nicht. ${ }^{4}$ So ist - um nur ein Beispiel zu erwähnen - in Bezug auf das Konstrukt der interkulturellen Kompetenz keineswegs geklärt, inwiefern ihre postulierten Teilkompetenzen in einem analytischen oder empirischen Zusammenhang zueinanderstehen (Straub 2007).

Im Unterschied zu Brandtstädter messen Holzkamp, Werbik und die mit ihm verknüpfte Erlanger Handlungspsychologie gerade auch als Konsequenz aus den angedeuteten wissenschaftstheoretischen und methodologischen Auseinandersetzungen experimentellen Verfahren und quantifizierenden Methoden in der Psychologie für handlungspsychologische Analysen eine vergleichsweise geringe Wichtigkeit zu. Für die Erlanger Handlungspsychologie kann wohl gesagt werden, dass in ihr analytischen Zusammenhängen insbesondere in Form von semantischen Verweisungszusammenhängen eine herausragende Bedeutung zukommt. Dabei steht nicht die Explikation von ,bloß“ begriffslogischen und semantischen, sondern die interpretative Rekonstruktion von semantischen, genauer: von pragma-semantischen (Weidemann 2018) Verweisungszusammenhängen in synchron wie diachron variablen und dynamischen soziokulturellen Kontexten im Zentrum ihrer Aufmerksamkeit. Oder noch einmal ein wenig anders und an einem simplifizierenden Beispiel kurz veranschaulicht: In dem einen Fall würde etwa danach gefragt werden, inwiefern der Begriff der Sorge nicht bereits unter anderem impliziere, dass man etwas erwarte, von dem man befürchte, es werde eintreten, von dem aber auch nicht gänzlich sicher sei, dass es eintreten werde und von dem man auch nicht sicher sei, inwiefern man es selbst werde (mit-)abwenden können. Eine möglichst genaue Explikation des Begriffs der Sorge könnte hier etwa die Voraussetzung für die Konstruktion eines Fragebogens sein, in dem einschlägige Items so formuliert und in Beziehung zueinander gesetzt würden, dass eben möglichst keine Verwechslung von semantischen und empirischen Zusammenhängen drohe. In dem anderen Fall läge das Interesse dagegen auf einer Rekonstruktion von in auf Sorge bezogene Handlungen und in sie eingelassene Bedeutungszusammenhänge in dem Bewusstsein, dass solche Handlungen und Bedeutungen relativ zu geschichtlichen - biografisch wie kollektiv - und kulturellen Kontexten unterschiedlich ausfallen können, mithin nicht invariant sind. Die dabei herausgearbeiteten mehr oder weniger komplexen und vielschichtigen Handlungen und Bedeutungen würden hier nicht als separat voneinander anzusehende Variablen betrachtet, sondern in ihren vielfältigen Verweisungen aufeinander analysiert werden.

Eingangs war festgehalten worden, dass die Erlanger Handlungspsychologie als am dortigen Institut für Psychologie verankerter Arbeitszusammenhang Geschichte sei. Die Auseinandersetzung mit historischen Phänomenen - im Hinblick auf die Psychologie, aber auch sonst - ist aber niemals eine bloße Auseinandersetzung mit der Vergangenheit, sondern stets auch eine mit der Gegenwart und der Zukunft. Mein Anliegen auf den vorangehenden Seiten war es zu zeigen, dass sowohl im Hinblick auf zentrale theoretische als auch methodologisch-methodische Fragen sowie in Bezug auf konkrete empirische Studien die Erlanger Handlungspsychologie nach

\footnotetext{
${ }^{4}$ Es scheint mir übrigens kein Zufall zu sein, dass eine der wenigen Einführungen in psychologische Forschungsmethoden, die sich dieser Thematik ernsthaft widmen, von dem ,ehemaligen Erlanger“ Wilhelm Kempf stammt (Kempf 2003; dazu: Kölbl 2005).
} 
wie vor Wesentliches zu sagen hat. Darüber hinaus bleibt anzumerken, dass aber auch außerhalb Erlangens und des dort angesiedelten Instituts für Psychologie seit langem an Weiterentwicklungen des handlungstheoretischen (und kulturpsychologischen) Ansatzes gearbeitet wird. Wie die Erlanger Handlungspsychologie fortlebt, in andere an sie anschließende, verwandte oder konkurrierende Forschungsprogramme eingemündet ist, kritisiert, ausdifferenziert oder modifiziert wurde, konnte und sollte hier jedoch nicht näher betrachtet werden. Diese insbesondere in Hannover, Amsterdam, Chemnitz, Zwickau, Bochum und Bayreuth fortgeschriebene Geschichte ,steht auf einem anderen Blatt“ bzw. gleich auf mehreren anderen Blättern und wäre Gegenstand eines gesonderten Beitrags.

Funding Open Access funding enabled and organized by Projekt DEAL.

Open Access Dieser Artikel wird unter der Creative Commons Namensnennung 4.0 International Lizenz veröffentlicht, welche die Nutzung, Vervielfältigung, Bearbeitung, Verbreitung und Wiedergabe in jeglichem Medium und Format erlaubt, sofern Sie den/die ursprünglichen Autor(en) und die Quelle ordnungsgemäß nennen, einen Link zur Creative Commons Lizenz beifügen und angeben, ob Änderungen vorgenommen wurden.

Die in diesem Artikel enthaltenen Bilder und sonstiges Drittmaterial unterliegen ebenfalls der genannten Creative Commons Lizenz, sofern sich aus der Abbildungslegende nichts anderes ergibt. Sofern das betreffende Material nicht unter der genannten Creative Commons Lizenz steht und die betreffende Handlung nicht nach gesetzlichen Vorschriften erlaubt ist, ist für die oben aufgeführten Weiterverwendungen des Materials die Einwilligung des jeweiligen Rechteinhabers einzuholen.

Weitere Details zur Lizenz entnehmen Sie bitte der Lizenzinformation auf http://creativecommons.org/ licenses/by/4.0/deed.de.

\section{Literatur}

Aschenbach, Günter. 1984. Erklären und Verstehen in der Psychologie. Zur methodischen Grundlegung einer humanistischen Psychologie. Bonn: Bock + Herchen.

Aschenbach, Günter, Elfriede Billmann-Mahecha, Jürgen Straub, und Hans Werbik. 1983. Das Problem der Konsensbildung und die Krise der ,nomothetischen“ Psychologie. In Psychologie in der Veränderung. Perspektiven für eine gegenstandsangemessenere Forschungspraxis, Hrsg. Gerd Jüttemann, 103-143. Weinheim: Beltz.

Billmann-Mahecha, Elfriede. 1994. Über die Interpretation von Kinderzeichnungen. L.O.G.O.S. interdisziplinär 2(1):28-35.

Billmann-Mahecha, Elfriede, und Hans-Jürgen Seel. 1981. Beratung im Forschungsprozess. In Konflikt und Konfliktbewältigung, Hrsg. Wilhelm Kempf, Günter Aschenbach, 367-397. Bern: Huber.

Boesch, Ernst E. 1991. Symbolic action theory and cultural psychology. Berlin, Heidelberg: Springer.

Boesch, Ernst E. 2002. Psychologie der Betroffenheit. Verhaltenstherapie \& Psychosoziale Praxis 34:329-331.

Boesch, Ernst E. 2021/2010. Kulturpsychologie. Rückblick und Plädoyer. In Musik, Sprache und die Sehnsucht nach dem Paradies. Ausgewählte Schriften zur Handlungs- und Kulturpsychologie, Hrsg. Jürgen Straub, 25-48. Gießen: Psychosozial-Verlag.

Brandtstädter, Jochen. 1982. Apriorische Elemente in psychologischen Forschungsprogrammen. Zeitschrift für Sozialpsychologie 13:267-277.

Brandtstädter, Jochen. 1984. Apriorische Elemente in psychologischen Forschungsprogrammen: Weiterführende Argumente und Beispiele. Zeitschrift für Sozialpsychologie 15:151-158.

Brandtstädter, Jochen. 2001. Entwicklung - Intentionalität - Handeln. Stuttgart: Kohlhammer.

Brandtstädter, Jochen, und Werner Greve. 1999. Intentionale und nichtintentionale Aspekte des Handelns. In Handlungstheorie. Begriff und Erklärung des Handelns im interdisziplinären Diskurs, Hrsg. Jürgen Straub, Hans Werbik, 185-212. Frankfurt/M.: Campus. 
Greve, Werner. 2001. Traps and gaps in action explanation. Theoretical problems of a psychology of human action. Psychological Review 108(2):435-451.

Groeben, Norbert, und Brigitte Scheele. 1979. Argumente für eine Psychologie des reflexiven Subjekts. Darmstadt: Steinkopff.

Heckhausen, Heinz. 1980. Motivation und Handeln. Lehrbuch der Motivationspsychologie. Berlin: Springer.

Holzkamp, Klaus. 1972. Kritische Psychologie. Vorbereitende Arbeiten. Frankfurt/M.: Fischer.

Holzkamp, Klaus. 1976. Zu diesem Heft. Zeitschrift für Sozialpsychologie 7:309.

Holzkamp, Klaus. 1986. Die Verkennung von Handlungsbegründungen als empirische Zusammenhangsannahmen in sozialpsychologischen Theorien. Methodologische Fehlorientierung infolge von Begriffsverwirrung. Zeitschrift für Sozialpsychologie 17:216-238.

Holzkamp, Klaus. 1993. Lernen. Subjektwissenschaftliche Grundlegung. Frankfurt/M.: Campus.

Holzkamp, Klaus. 1996. Über die Entstehung und Entwicklung der Kritischen Psychologie. Ein Interview. Teil I und Teil II. Das Argument 215/216:405-414/565-580.

Kaiser, Heinz Jürgen, und Hans Werbik. 1977. Der „Telefonzellenversuch“. Ein erstes Experiment zur Überprüfung einer Theorie sozialen Handelns. Zeitschrift für Sozialpsychologie 8:115-129.

Kaiser, Heinz Jürgen, und Hans Werbik. 2012. Handlungspsychologie. Eine Einführung. Göttingen: Vandenhoeck \& Ruprecht.

Kaiser, Heinz Jürgen, und Hans Werbik. 2018. Handlung. In Stichwörter zur Kulturpsychologie, Hrsg. Carlos Kölbl, Anna Sieben, 181-187. Gießen: Psychosozial-Verlag.

Kamlah, Wilhelm. 1972. Philosophie Anthropologie. Mannheim: Bibliographisches Institut.

Kamlah, Wilhelm, und Paul Lorenzen. 1967. Logische Propädeutik. Vorschule des vernünftigen Redens. Mannheim: Bibliographisches Institut.

Kempf, Wilhelm. 1978. Konfliktlösung und Aggression. Zu den Grundlagen einer psychologischen Friedensforschung. Bern: Huber.

Kempf, Wilhelm. 2003. Theorie und Empirie. Forschungsmethoden der Psychologie. Zwischen naturwissenschaftlichem Experiment und sozialwissenschaftlicher Hermeneutik, Bd. 1. Berlin: Regener.

Kochinka, Alexander. 2020. Symbolische Handlungstheorie. In Psychologie der Polyvalenz. Ernst Boeschs Kulturpsychologie in der Diskussion, Hrsg. Jürgen Straub, Pradeep Chakkarath, und Sebastian Salzmann, 169-190. Bochum: Westdeutscher Universitätsverlag.

Kölbl, Carlos. 2003. Das aktionale Entwicklungsparadigma jenseits subjektseitiger Allmachtsansprüche. Handlung Kultur Interpretation 12(1):181-189.

Kölbl, Carlos. 2005. Rezension zu Wilhelm Kempf: Forschungsmethoden der Psychologie. Forum Qualitative Sozialforschung https://doi.org/10.17169/fqs-6.2.486.

Kölbl, Carlos. 2006. Development as enculturation and active formation revisited. In Pursuit of meaning. Advances in cultural and cross-cultural psychology, Hrsg. Jürgen Straub, Doris Weidemann, Carlos Kölbl, und Barbara Zielke, 495-513. Bielefeld: transcript.

Kölbl, Carlos. 2020a. Kulturpsychologie der Erziehung und Bildung. Ernst Boesch als Pädagogischer Psychologe. In Psychologie der Polyvalenz. Ernst Boeschs Kulturpsychologie in der Diskussion, Hrsg. Jürgen Straub, Pradeep Chakkarath, und Sebastian Salzmann, 213-271. Bochum: Westdeutscher Verlag.

Kölbl, Carlos. 2020b. Ernst Boesch's cultural psychology of education. Culture \& Psychology 26(2): $159-172$.

Korthals-Beyerlein, Gabriele. 1979. Soziale Normen. Begriffliche Explikation und Grundlagen empirischer Erfassung. München: Fink.

Lenk, Hans (Hrsg.). 1977-1984. Handlungstheorien interdisziplinär I-IV. München: Fink.

Lerner, Richard M., und Nancy A. Busch-Rossnagel (Hrsg.). 1981. Individuals as producers of their development. A life-span perspective. New York: Academic Press.

Lorenzen, Paul, und Oswald Schwemmer. 1975. Konstruktive Logik, Ethik und Wissenschaftstheorie. Mannheim: Bibliographisches Institut.

Popp, Ulrike. 1989. Mythen und Motive autoritären Handelns. Ein kulturpsychologischer Beitrag zur Autoritarismusforschung. Frankfurt/M.: Campus.

Smedslund, Jan. 1991. Psycho-logic. New York: Springer.

Straub, Jürgen. 1999. Handlung, Interpretation, Kritik. Grundzüge einer textwissenschaftlichen Handlungs- und Kulturpsychologie. Berlin, New York: deGruyter.

Straub, Jürgen. 2007. Kompetenz. In Handbuch interkulturelle Kommunikation und Kompetenz, Hrsg. Jürgen Straub, Arne Weidemann, und Doris Weidemann, 35-46. Stuttgart: Metzler.

Straub, Jürgen. 2020. Handlungstheorie. In Handbuch Qualitative Forschung in der Psychologie, Hrsg. Günter Mey, Katja Mruck, 263-282. Berlin: Springer. 
Straub, Jürgen. 2021a. Die wahre Geschichte eines Schweizers, der auszog das Forschen zu lernen: Ernst Boeschs Psychologie und relationale Hermeneutik sowie die vertrackte Frage nach der Methode. Vortrag auf der gemeinsamen Tagung der Gesellschaft für Kulturpsychologie und der DGPs-Fachgruppe Geschichte der Psychologie, Monte Verità Ascona, 23.9.21.

Straub, Jürgen. 2021b. Psychologie als interpretative Sozial- und Kulturwissenschaft. Menschenbild, Wissenschaftsverständnis und theoretische Programmatik. Gießen: Psychosozial-Verlag.

Straub, Jürgen. 2022. Verstehendes Erklären. Methodologie und Methodik einer relationalen Hermeneutik. Schriften zu einer handlungstheoretischen Kulturpsychologie. Gießen: Psychosozial-Verlag (im Druck).

Straub, Jürgen, Hans Werbik, und Walter Zitterbarth. 1987. Friedensbewegung und Kriegsängste. Über einige Aspekte der motivationalen Hintergründe friedenspolitischer Aktivitäten. In Mit Kriegsgefahren leben. Bedrohtsein, Bedrohungsgefühle und friedenspolitisches Engagement, Hrsg. Klaus Horn, Volker Rittberger. Opladen: Westdeutscher Verlag.

Thomas, Alexander (Hrsg.). 1976. Psychologie der Handlung und Bewegung. Meisenheim, Glan: Hain.

Toebe, Peter, Jochen Harnatt, Oswald Schwemmer, und Hans Werbik. 1977. Beiträge der konstruktiven Philosophie zur Klärung der begrifflichen und methodischen Grundlagen der Psychologie. In Wissenschaftstheoretische Grundlagen der Psychologie, Hrsg. Klaus A. Schneewind, 93-115. München: Reinhardt.

Vorlesungsverzeichnisse der Friedrich-Alexander-Universität Erlangen Nürnberg, Sommersemester 1979 bis Wintersemester 2005/6. Erlangen: Universität Erlangen-Nürnberg.

Weidemann, Arne. 2018. Pragma-Semantik. In Stichwörter zur Kulturpsychologie, Hrsg. Carlos Kölbl, Anna Sieben, 315-320. Gießen: Psychosozial-Verlag.

Werbik, Hans. 1971. Das Problem der Definition aggressiver Verhaltensweisen. Zeitschrift für Sozialpsychologie 2:233-247.

Werbik, Hans. 1974. Theorie der Gewalt. München: Fink.

Werbik, Hans. 1976a. Grundlagen einer Theorie sozialen Handelns. Teil 1: Aufbau der handlungstheoretischen Terminologie. Zeitschrift für Sozialpsychologie 7:248-261.

Werbik, Hans. 1976b. Grundlagen einer Theorie sozialen Handelns. Teil 2: Regeln für die Entwicklung empirischer Hypothesen. Zeitschrift für Sozialpsychologie 7:310-326.

Werbik, Hans. 1978. Handlungstheorien. Stuttgart: Kohlhammer.

Werbik, Hans. 1984. Über die nomologische Auslegung von Handlungstheorien. In Verhaltenswissenschaftliche und psychologische Handlungstheorien. Zweiter Halbband. Handlungstheorien interdsziplinär, Bd. III, Hrsg. Hans Lenk, 633-651. München: Fink.

Werbik, Hans. 1985a. „Psychonomie“ und „Psychologie“. Zur Notwendigkeit der Unterscheidung zweier Wissenschaften. In Technische Rationalität und rationale Heuristik, Hrsg. Clemens Burrichter, Rüdiger Inhetveen, und Rudolf Kötter, 109-121. Paderborn: Schöningh.

Werbik, Hans. 1985b. Die Versuchspersonen werden vergebens getäuscht. Zur Überflüssigkeit experimenteller Methoden in der Sozialpsychologie. In Humane Experimente? Genbiologie und Psychologie, Hrsg. Hans Lenk, 149-151. München: Fink.

Werbik, Hans. 1986. Existenz-Psychologie. Memorandum, Bd. Nummer 22. Erlangen-Nürnberg: Institut für Psychologie der Friedrich-Alexander-Universität Erlangen-Nürnberg. 1987 unter demselben Titel im von Manfred Amelang herausgegebenen Band 2 des Kongresses der Deutschen Gesellschaft für Psychologie in Heidelberg abgedruckt.

Werbik, Hans. 1987. Zur rationalen Annehmbarkeit handlungspsychologischer Aussagen und TheorieSkizzen. In Struktur und Erfahrung in der psychologischen Forschung, Hrsg. Jochen Brandtstädter, 125-158. Berlin, New York: deGruyter.

Werbik, Hans, und Heinz-Jürgen Kaiser. 1981. Vorwort. In Kritische Stichwörter zur Sozialpsychologie, Hrsg. Hans Werbik, Heinz-Jürgen Kaiser, 7-13. München: Fink.

Zielke, Barbara. 2004. Kognition und soziale Praxis. Der soziale Konstruktionismus und die Perspektiven einer postkognitivistischen Psychologie. Bielefeld: transcript.

Zitterbarth, Walter. 1988. Kulturpsychologie. In Handwörterbuch der Psychologie, 4. Aufl., Hrsg. Roland Asanger, Gerd Wenninger, 382-386. München, Weinheim: PVU.

Zitterbarth, Walter, und Hans Werbik. 1990. Die „Halbherzigkeit“ der kognitiven Wende. In Wissenschaftsund Erkenntnistheorie. Eine Einführung für Psychologen und Humanwissenschaftler, 2. Aufl., Hrsg. Jürgen Kriz, Helmut Lück, und Horst Heidbrink, 229-243. Opladen: Leske + Budrich.

Hinweis des Verlags Der Verlag bleibt in Hinblick auf geografische Zuordnungen und Gebietsbezeichnungen in veröffentlichten Karten und Institutsadressen neutral. 